\title{
Disfunção temporomandibular na visão de profissionais e acadêmicos de odontologia
}

\author{
Temporomandibular disorders from the perspective \\ of dentistry academics and professionals
}

\author{
Adriana Cristina ZAVANELLI ${ }^{\prime}$ \\ Paulo Renato Junqueira ZUIM \\ Gláucia de Souza BARBOZA² \\ Mirella Martins JUSTI ${ }^{2}$
}

\begin{abstract}
Resumo
Este artigo apresenta os resultados de uma pesquisa qualitativa aplicada a alunos e profissionais de Odontologia que trabalham com pessoas que sofrem de disfunção temporomandibular. Objetivou-se investigar a forma como os profissionais e acadêmicos de Odontologia percebem seus pacientes acometidos por essa disfunção, seus sentimentos diante do sofrimento dos mesmos e a importância de uma abordagem multidisciplinar, pontuando quais os tratamentos utilizados. Vale ressaltar que, na problemática da disfunção temporomandibular, estão envolvidos vários fatores, dentre eles o emocional, citado pela maioria dos entrevistados. Os dados obtidos em relação ao conhecimento dos profissionais e alunos quanto ao atendimento do paciente com disfunção temporomandibular foi considerado adequado; entretanto, mostraram inadequações importantes, como o fato de os profissionais não investigarem melhor o histórico de vida do paciente em seu aspecto psicossocial.
\end{abstract}

Unitermos: Articulação temporomandibular; Odontologia; Tratamento multidisciplinar.

\begin{abstract}
This paper presents the results of an applied qualitative study with dental students and professionals who work with people suffering from temporomandibular disorder. The aim was to investigate the way that dental practitioners and students perceive patients affected by this disorder, how they feel faced with the patients' suffering, and the importance they assign to a multidisciplinary approach, highlighting the treatments used. It is noteworthy that various factors are involved in the temporomandibular disorder framework, including emotional factors, as cited by the majority of the respondents. The data obtained indicated that the knowledge of the professionals and students related to caring for people with temporomandibular disorders was appropriate, however, important inadequacies were highlighted, such as the fact that professionals do not investigate the life history of the person in their psychosocial context.
\end{abstract}

Uniterms: Temporomandibular joint; Dentistry; Multidisciplinary treatment.

\footnotetext{
$\boldsymbol{\nabla} \nabla \nabla$
}

1 Universidade Estadual Paulista Júlio de Mesquita Filho, Faculdade de Odontologia de Araçatuba, Departamento de Materiais Odontológicos e Prótese. R. José Bonifácio, 1193, Vila Mendonça, 16015-050, Araçatuba, SP, Brasil. Correspondência para/Correspondence to: A. C. ZAVANELLI. E-mail: $<$ zavanelliac@foa.unesp.br>.

2 Universidade Estadual Paulista Júlio de Mesquita Filho, Faculdade de Odontologia de Araçatuba, Centro de Promoção da Qualidade de Vida. Araçatuba, SP, Brasil. 
Um dos primeiros a tentar explicar o mecanismo da dor foi Aristóteles. Ele dizia que a dor era uma emoção, e não uma sensação, desconsiderando possíveis fatores fisiológicos. Por volta de 1644, Descartes elaborou a Teoria da Especificidade da Dor, explicando que, para cada estímulo de dor, existiria um receptor específico, com uma via de ligação única, que seria traduzida quando essa informação chegasse a um centro de dor no sistema nervoso central. Assim, essa ideia de que a sensação de dor dependeria de uma lesão influenciou e ainda influencia profissionais na compreensão e no significado da dor (Garcia, 2006).

A partir do século $X X$, mais precisamente em 1965, com Melzack e Wall, a dor passou a ser vista como uma sensação e uma emoção. Esses autores afirmaram que a dor seria resultante da ligação entre fatores físicos, emocionais e cognitivos, surgindo, então, a Teoria da Comporta da Dor. Tal teoria refere-se à ideia de que a dor é regulada por uma "comporta" que pode se abrir ou se fechar por meio de impulsos derivados de nervos periféricos ou do sistema nervoso central, diminuindo ou aumentando a dor percebida (Nogueira, Bergmann \& Ribeiro, 2005). Tal constatação motivou o retorno à consideração de que os fatores psicológicos, juntamente com os fatores fisiológicos, existem na sensação de dor.

Nos dias atuais, de acordo com Guimarães (1999), a dor é considerada um problema biopsicossocial de difícil compreensão, pois envolve as diferentes dimensões humanas, dificultando sua mensuração. Uma das definições mais utilizadas nas pesquisas da área é a da International Association for the Study of Pain (IASP), que entende a dor como "uma experiência desagradável, sensitiva e emocional, associada a uma lesão real ou potencial dos tecidos ou descrita em termos dessa lesão" (Cruz, Alchieri \& Sarda Júnior, 2002, p.173).

Para Pessini (2009), a dor é fisiológica e o sofrimento, psicológico, sendo este segundo muito mais amplo do que o primeiro. $\mathrm{O}$ autor complementa essa ideia dizendo que a dor é uma experiência física e psíquica, e que a dor e o sofrimento se reforçam mutuamente: uma dor muito forte e constante pode influenciar todas as dimensões do sofrimento e, no sentido oposto, a ansiedade, a depressão, a solidão ou o sofrimento do vazio existencial podem acentuar a dor. Marquez (2011), usando uma visão humanista, diz que a"dor é o que o paciente diz ser e existe quando ele diz existir" (p.29), sugerindo que a dor é uma experiência particular a cada indivíduo.

Para a dor existem diversas classificações, mas, de maneira geral, os pesquisadores a dividem em dor aguda, aguda recorrente e crônica (Straub, 2005). Este estudo concentra-se na dor crônica por ser ela parte da sintomatologia referida nas Disfunções Temporomandibulares (DTM).

A dor crônica pode ter origens em doenças estruturais ou funcionais, permanecendo após a cura da lesão nos tecidos (Murta, 1999). Essa ideia abre várias questões por se tratar de um fenômeno em que, muitas vezes, não há um comprometimento físico da pessoa que justifique sentir tanta dor. Em muitos casos, a lesão é mínima ou até inexistente. Cardoso e Paraboni (2010), explicam que a instalação da dor localizada em uma determinada parte do corpo poderia corresponder a uma tentativa de controlar a "excitação traumática" criando uma forma de identificar e localizar a dor para sua melhor compreensão. Murta (1999) ressalta que há diversos pesquisadores discutindo a multiplicidade de fatores envolvidos no mecanismo da dor crônica; assim, têm sido adotados diferentes critérios e sistemas de classificação para o problema.

Segundo o Diagnostic and Statistical Manual of Mental Desorders (Murta, 1999), a dor pode ser considerada crônica após seis meses de duração, sendo classificada de três maneiras: (1) "desordem de dor associada com fatores psicológicos" - quando fatores psicológicos podem ser responsáveis pelo surgimento, exacerbação, severidade ou manutenção da dor; (2) "desordem de dor associada a fatores psicológicos e condição médica geral" - quando se entende que fatores psicológicos e condições médicas gerais podem estar relacionadas ao surgimento, exacerbação, severidade ou manutenção da dor; (3) "desordem de dor associada com condição médica geral" - quando a dor é resultante de condições médicas, não tendo fatores psicológicos associados ou sendo esses fatores psicológicos mínimos, a ponto de não interferirem no surgimento e na manutenção da dor. 
Atualmente, segundo Miceli (2002), a dor crônica é uma das principais causas da procura da população pelo sistema de saúde, pois acomete cerca de 30\% a 40\% da população brasileira, sendo uma das principais causas que levam o sujeito a faltar ao trabalho e a solicitar licenças médicas, indenizações trabalhistas, aposentadorias por doença e baixa produtividade. Esse fenômeno é considerado, de acordo com Silva e Zago. (2001), um fenômeno multifatorial e complexo, ligado a fatores físicos e emocionais.

A dor orofacial é denominada, segundo Siqueira e Teixeira (2001), um conjunto de condições dolorosas provenientes da boca e da face, não exclusiva da área odontológica. Inclui a dor de dente, as disfunções temporomandibulares, neuralgias, alguns tipos de cefaleias e outros quadros dolorosos.

A disfunção temporomandibular é o termo usado para explicar as várias condições clínicas que atingem os músculos mastigatórios. A Articulação Temporomandibular (ATM) e as estruturas associadas são consideradas a causa mais comum das dores orofaciais (Fujarra, 2008). Apesar dos vários estudos referentes à dor da DTM, são poucas as explicações sobre sua etiologia, mecanismos e tratamentos, conforme Hilgenberg (2009). A DTM apresenta características multifatoriais e seu tratamento deve ser multiprofissional. Ela tem como sintomas: dor na mandíbula e na região da ATM, dores de cabeça, ruído na articulação, dificuldade em abrir e fechar a boca, dificuldade em morder e cortar alimentos, e pode ocorrer quando a boca está em repouso, não ameaçando a vida da pessoa, mas gerando sofrimento (Bove, Guimarães \& Smith, 2005).

Dentre as desordens que apresentam manifestações de dor, a DTM e a dor orofacial são as que mais se mostram prevalentes na população. De acordo com Bove et al. (2005), 86\% da população ocidental apresenta sinais e sintomas de DTM ou dor orofacial, podendo ocorrer em qualquer idade, com prevalência em indivíduos com idade ente 13 e 35 anos e incidindo quatro vezes mais em mulheres.

Frente às definições e reflexões expostas sobre os processos de dor, mais especificamente da DTM e da dor orofacial, cabe agora o questionamento acerca de como os profissionais cirurgiões-dentistas e acadêmicos percebem esses processos. A percepção reflete na escolha dos temas de pesquisa, que podem resultar em avanços no conhecimento e estimulam o desenvolvimento de novas formas de tratamento.

\section{Os profissionais da área odontológica}

A presença de fatores emocionais relacionados à origem e manutenção de quadros como as DTM é citada por profissionais de várias áreas da saúde. Apesar de serem conhecidos os problemas causados pelas questões emocionais na vida das pessoas que sofrem com dor orofacial, nota-se que a maioria não adere ao tratamento proposto, principalmente o tratamento psicológico. Essa resistência em aceitar a presença de conteúdos emocionais na origem das doenças é compartilhada também por alguns profissionais da área clínica, que priorizam o tratamento físico para tentar aliviar o sofrimento. Atitudes como esta podem explicar casos de insucesso no tratamento da doença (Bérzin, 2007).

A compreensão das relações mente-corpo, objeto de estudos da psicossomática desde a década de 1930 (Alexander, 1989; Vasconcellos, 1998), foi fortalecida por resultados de pesquisas psicofisiológicas no campo do stress e, posteriormente, por estudos realizados no modelo psiconeuroendocrinoimunológico (Neme, Soliva \& Ribeiro, 2003; Vasconcellos, 2002), ampliando as possibilidades explicativas e interpretativas do fenômeno saúde-doença. O trabalho interdisciplinar tem sido valorizado em diferentes estudos, sendo apontado como aspecto de relevância científica.

De acordo com Vasconcellos (2000), a interface entre campos do conhecimento humano é de vital importância para a área da saúde, especialmente para as próximas décadas. Segundo o autor, sem o exercício da interdisciplinaridade será fomentada uma profunda iatrogenia e os profissionais serão responsáveis pelo descrédito e malefício não apenas do atendimento, mas de suas profissões.

Do ponto de vista social, este estudo, em área de interface entre a Psicologia e a Odontologia, é bastante relevante, visto que busca esclarecer aspectos psicossociais de pacientes com uma doença odontológica e avaliar benefícios à saúde física e mental dos pacientes, obtidos por meio de intervenções psico- 
lógicas e de psicoterapia breve, fortalecendo a interdisciplinaridade e a cooperação entre cirurgiões-dentistas e psicólogos.

Os resultados obtidos permitem indicar a psicoterapia como importante forma de tratamento coadjuvante em casos de doenças orgânicas que ainda não dispõem de tratamentos medicamentosos efetivos e para quadros orgânicos que demonstrem melhoras com o auxílio da psicoterapia. Femiano, Gombos e Scully (2004) utilizaram e relataram a eficácia da psicoterapia psicanalítica na Síndrome da Ardência Bucal, enfatizando que esse método poderia ser estendido a outras enfermidades.

No caso das psicodermatoses, a literatura ressalta a necessidade de auxílio psicoterápico em diferentes doenças dermatológicas, especialmente as que têm suas gêneses pouco esclarecidas e para as quais não se dispõe ainda de tratamentos medicamentosos eficientes (Azevedo, 2007; Hoffmann, Zogobi, Fleck \& Muller, 2005; Prado \& Neme, 2008).

Com relação à interface Psicologia e Odontologia, Seger (2002) indica os benefícios da Terapia Centrada na Disfunção (TCD) para pacientes com disfunção da articulação temporomandibular e a necessidade dos tratamentos psicológicos coadjuvantes no tratamento da Gengivite Ulcerativa Necrotizante (GUN) e de outras patologias bucais, assim como nas cirurgias odontológicas, nas lesões labiopalatinas, na colocação de próteses e na ortodontia.

Os resultados encontrados na literatura em psicossomática e na área da psicoimunologia são indicativos da integração psicofisiológica na saúde e na doença, fortalecendo a utilização de técnicas e recursos psicológicos e psicoterapêuticos na promoção, prevenção e tratamento das moléstias orgânicas (Mello Filho, 1992).

Cada vez mais se constata a relevância da colaboração entre cirurgiões-dentistas e psicólogos (Seger, 2002), tanto na investigação científica de fenômenos de interesse para ambas as áreas, como na intervenção junto a portadores de patologias cuja gênese indica a junção de fatores biopsicossociais e cujo tratamento médico, odontológico e medicamentoso não é curativo, não se dispondo, em muitos casos, de condutas médico-odontológicas totalmente satisfatórias.
A formação profissional na área de dor é referência na literatura, pois o profissional precisa conhecer a doença para realizar um diagnóstico correto e, assim, conhecer melhor a terapêutica a ser usada no tratamento da dor (Bérzin, 2007).

É importante também que o profissional conheça melhor o paciente em termos biológicos, psicológicos e sociais, compreendendo as suas experiências, seu comportamento e o seu padrão de qualidade de vida (Bérzin, 2007).

Ainda hoje muitos profissionais da saúde não levam em consideração os fatores emocionais relacionados ao problema da dor, mesmo não encontrando fatores biológicos que expliquem tal problema (Gameiro, Andrade, Nouer \& Veiga, 2006). Apesar dessa dificuldade com os profissionais, pesquisas comprovam a eficácia dos tratamentos psicológicos nos casos de dor, mostrando que não há linha divisória nítida para o tratamento físico e o não físico (Straub, 2005).

Levando em consideração o abrangente universo de pesquisas sobre a dor orofacial, como a população vem sendo acometida pela doença e sua significância para a saúde pública, o objetivo dessa pesquisa foi investigar a visão que os profissionais cirurgiões-dentistas fazem do paciente com disfunção temporomandibular, como eles se sentem lidando com a dor de seu paciente, qual a importância de um tratamento multiprofissional no caso da disfunção temporomandibular e quais os tratamentos mais utilizados, buscando dados que mostrem como são realizados atualmente os tratamentos da dor.

Assim, pretendeu-se entender melhor a relação estabelecida entre profissional/paciente a partir do relato dos cirurgiões-dentistas em relação aos atendimentos que realizam.

\section{Procedimentos}

O estudo foi aprovado pelo Comitê de Ética em Pesquisa da Universidade Estadual Paulista Júlio de Mesquita Filho (Unesp), Faculdade de Odontologia, campus Araçatuba (SP), Processo no FOA 01249/2010, em 15/9/2010, e todos os participantes assinaram um Termo de Consentimento Livre e Esclarecido antes de sua inclusão na amostra. 
As entrevistas foram realizadas com nove profissionais, graduandos e pós-graduandos do Núcleo de Diagnóstico e Tratamento das Disfunções Temporomandibulares (NDTDTM) que trabalham com dor orofacial crônica na Unesp, em Araçatuba (SP). Por se tratar de poucos profissionais - alunos de graduação e pós-graduandos que atuam neste núcleo -, o trabalho foi realizado com todos que aceitaram participar da pesquisa.

As entrevistas foram gravadas em áudio e posteriormente transcritas na íntegra; logo após, foram lidas várias vezes, buscando-se obter as categorias de significado das informações coletadas na fala dos entrevistados.

\section{Resultados e Discussão}

Foram realizadas nove entrevistas com estudantes e profissionais da área de Odontologia, sendo três alunos do quarto ano de Odontologia e seis da Especialização em Prótese Dentária. Todos prestavam assistência ao Núcleo de Diagnóstico e Tratamento das Disfunções Temporomandibulares (NDTDTM), na Unesp de Araçatuba (SP).

As entrevistas continham perguntas acerca da definição de alunos e profissionais da Odontologia sobre pacientes com dor, como eles se sentiam ao lidar com a pessoa em sofrimento e qual a relevância dada por eles ao tratamento multiprofissional.

Primeiro, quando solicitados a expressar em poucas palavras sua definição do paciente com dor, os participantes revelaram uma variedade de significados, dentre eles o de que a pessoa com dor é depressiva, angustiada, alguém que precisa de ajuda, uma pessoa que tem medo, com problemas sociais, com problemas psicológicos, que perdeu alguém importante, que não aparenta estar de bem com a vida, desesperada de dor, que pede urgência, paciente diferenciado, que gosta de falar. Aparentemente, os entrevistados descreveram seus pacientes com dor de forma generalizada, sem um conceito bem definido sobre o problema: apenas duas pessoas apontaram a mesma descrição para esse tipo de paciente.

A segunda pergunta investigava como o profissional se sentia diante do sofrimento de seu paciente.
Os participantes, em sua maioria, mostraram-se sensibilizados com o sofrimento do paciente com dor orofacial crônica, exceto os casos em que o não envolvimento foi citado (dois casos). Entre as sensações e os sentimentos descritos estão: angústia, perda de apetite, motivação para ajudar, paciência, limitação e descontentamento quando o tratamento não tem efeito.

Ao comparar as respostas dos alunos do quarto ano de Odontologia com as respostas dos pós-graduandos, foi possível perceber que, para os alunos do quarto ano, os atendimentos pareciam afetá-los mais diretamente - por exemplo, quando explicitaram seus sentimentos por meio de sensações corporais que percebiam depois do atendimento realizado à pessoa com dor. Enquanto os alunos da especialização aparentaram não se envolver tanto, os de graduação comentaram que se solidarizam, tentam ajudar; a fala, no entanto, é aparentemente mais fria, sendo perceptível a presença de mecanismos defensivos provavelmente aprendidos com a práxis.

Ao tratar da questão da importância do tratamento multiprofissional para a pessoa com dor, a maioria dos entrevistados apontou ser fundamental - especialmente para aquele com dor orofacial crônica. Todos os entrevistados concordaram sobre a necessidade de outros profissionais estarem envolvidos no tratamento da dor; entre os profissionais citados, estão o psicólogo e o fisioterapeuta.

De modo geral, os dados coletados são positivos com relação ao conhecimento dos profissionais quanto ao atendimento do paciente com DTM. O que falta, no entanto, para que esse atendimento seja completo? As entrevistas revelaram o fato de que os profissionais não investigam o histórico de vida da pessoa em seu aspecto psicossocial; fazem um amplo levantamento biológico, mas não buscam outras informações que também fazem parte da constituição do indivíduo, ou seja, os aspectos psicológicos e sociais que podem estar envolvidos no surgimento e manutenção da dor. As entrevistas mostraram, por outro lado, que os profissionais já percebem a importância das práticas humanizantes e multiprofissionais.

É preciso ir além do que está posto, ou seja, não se pode enfatizar apenas o aspecto biológico, mas analisar o indivíduo em sua totalidade, pois há um conjunto de fatores envolvidos no surgimento da DTM. Essa 
investigação sobre os aspectos biopsicossociais poderia ser realizada por uma equipe interdisciplinar, que levantaria todos os fatores que podem envolver o surgimento da doença e depois promoveria a discussão entre os profissionais; assim, o tratamento poderia obter o resultado esperado.

Segundo Gameiro (2006), ainda hoje muitos profissionais da saúde não levam em consideração os fatores emocionais relacionados ao problema da dor, mesmo não encontrando fatores biológicos que expliquem tal problema. Apesar dessa dificuldade com os profissionais, pesquisas comprovam a eficácia dos tratamentos psicológicos nos casos de dor, mostrando que não há linha divisória nítida para o tratamento físico e o não físico (Straub, 2005). Dessa forma, seria importante uma pesquisa que buscasse avaliar as grades curriculares das universidades, pois essa resistência pode estar associada à formação dos profissionais da área odontológica. Se houver uma reforma nas grades acadêmicas que contemple uma visão biopsicossocial, poderá haver uma mudança significativa no olhar desses profissionais.

A dor orofacial crônica é considerada um problema de saúde pública que acomete grande parte da população, sendo um problema complexo e de difícil tratamento, por envolver vários fatores na origem e na manutenção da doença.

Os entrevistados, em sua maioria, não apresentaram dificuldades em falar de seus pacientes. Alguns se esquivaram quando foi solicitado que falassem de seus sentimentos diante do sofrimento do paciente, mas conseguiram responder de forma geral. O trabalho multiprofissional foi considerado muito importante para o tratamento das pessoas com dor.

A visão que os profissionais cirurgiões-dentistas fazem hoje das pessoas com DTM revela que são identificados fatores emocionais que podem estar envolvidos no surgimento da dor, como no caso de cinco dos nove entrevistados nessa pesquisa, que mencionaram ser necessário o acompanhamento psicológico no tratamento da dor crônica.

A pesquisa mostrou que seria de grande relevância uma maior investigação do histórico biopsicossocial dos pacientes, sugerindo-se, assim, a necessidade de uma equipe interdisciplinar que possa fazer esse levantamento, proporcionando uma visão do todo e norteando o tratamento da dor.

\section{Referências}

Alexander, F. (1989). Medicina psicossomática: princípios e aplicações. Porto Alegre: Artes Médicas.

Azevedo, G. M. G. (2007). A criança com psoríase e as relações vinculares com a mãe e a família (Dissertação de mestrado não-publicada). Programa de Pós-Graduação em Psicologia do Desenvolvimento e Aprendizagem, Universidade Estadual Paulista, Bauru.

Bérzin, M. G. R. (2007). Características da formação profissional: prática clínica e perfil biopsicossocial de cirurgiões-dentistas e médicos que atuam na área de dor orofacial (Tese de doutorado não-publicada). Programa de Pós-Graduação em Biologia Buco-Dental, Universidade Estadual de Campinas.

Bove, S. R. K, Guimarães, A. S., \& Smith, R. L. (2005). Caracterização dos pacientes de um ambulatório de disfunção temporomandibular e dor orofacial. Revista Latino-Americana de Enfermagem, 13(5), 686-691. Recuperado em novembro 3, 2010, disponível em <www.scielo.br/rlae>.

Cardoso, M. R., \& Paraboni, P. (2010). Dor física crônica: uma estratégia de sobrevivência psíquica? Revista Mal-Estar e Subjetividade, 10(4), 1203-1220. Recuperado em dezembro 19, 2011, disponível em <www.pepsic.bvsalud.org>.

Cruz, R. M, Alchieri J. C., \& Sarda Júnior, J. J. (2002). Avaliação e medidas psicológicas: produção do conhecimento e da intervenção profissional. São Paulo: Casa do Psicólogo.

Femiano, F., Gombos, F., \& Scully, C. (2004). Síndrome de boca ardiente: estúdio de la psicoterapia, medicación com ácido alfa-lipoico y combinación de terapias. Medicina Oral y Cirurgía Bucal, 9(1), 8-13.

Fujarra, F. J. C. (2008). Disfunção temporomandibular e síndrome fibromiálgica: caracterização de amostra segundo critérios clínicos (Dissertação de mestrado não-publicada). Programa de Pós-Graduação em Neurologia, Universidade de São Paulo.

Gameiro G. H, Andrade A. S., Nouer D. F., \& Veiga, M. C. F. A. (2006). How may stressful experiences contribute to the development of temporomandibular disorders? Clinical Oral Investigations, 10(4), 261-268.

Garcia, D. M. (2006). Crenças de profissionais e centros de dor sobre dor crônica (Dissertação de mestrado não-publicada). Programa de Pós-Graduação em Enfermagem, Universidade de São Paulo.

Guimarães, S. S. (1999). Introdução ao estudo da dor. In M. M. M. J. (Org.), Dor: um estudo multidisciplinar (pp.13-30). São Paulo: Summus.

Hilgenberg, P. B. (2009). Estudo da participação de sinais e sintomas de disfunção temporomandibular (DTM) e sintomas otológicos em pacientes portadores de zumbido subjetivo (Dissertação de mestrado não-publicada). Programa de Pós-Graduação em Ciências Odontológicas Aplicadas, Universidade de São Paulo.

Hoffmann, F. S. Zogbi, H., Fleck, P., \& Muller, M. C. (2005). A integração mente e corpo em psicodermatologia. Psicologia: Teoria e Prática, 7(1), 51-60.

Marquez, J. O. (2011). A dor e os seus aspectos multidimensionais. Ciência e Cultura, 63(2), 28-32. Recuperado 
em dezembro 19, 2011, disponível em <www.cienciae cultura.bvs.br>.

Mello Filho, J. (1992). Psicossomática hoje. Porto Alegre: Artes Medicas.

Miceli, A. V. P. (2002). Dor crônica e subjetividade em oncologia. Revista Brasileira de Cancerologia, 48(3), 363-373.

Murta, S. G. (1999). Avaliação e manejo da dor crônica. In M. M. M. J. Carvalho (Org.), Dor: um estudo multidisciplinar (2a ed., pp.174-195). São Paulo: Summus.

Neme, C. M. B., Soliva, S. N., \& Ribeiro, E. J. (2003). História prévia de eventos de estresse e câncer de mama, útero e ovários. In C. M. B. Neme \& O. M. P. R. Rodrigues (Orgs.), Psicologia dasaúde:perspectivas interdisciplinares (pp.95-124). São Carlos: Rima.

Nogueira, E. A., Bergmann, A., \& Ribeiro, M. J. P. (2005). Abordagem fisioterapêutica na mama fantasma em mulheres submetidas à cirurgia de mastectomia. Revista FisioBrasil, $72,43-48$.

Pessini, L. (2009). Humanização da dor e sofrimento humanos no contexto hospitalar. Revista Bioética, 10(2), 51-72.

Prado, R. B. R., \& Neme, C. M. B. (2008). Experiências afetivo-familiares de mulheres com alopecia areata. Estudos de Psicologia (Campinas), 25(4), 487-498. doi: 10.1590/S010 3-166×2008000400016.
Seger, L. (2002). Psicologia e odontologia: uma abordagem integradora. São Paulo: Livraria e Editora Santos.

Silva, L. M. H., \& Zago, M. M. F. (2001). O Cuidado do paciente oncológico com dor crônica na ótica do enfermeiro. Revista Latino-Americana de Enfermagem, 9(4), 44-49. Recuperado em novembro 13, 2010, disponível em $<$ www.scielo.br/rlae $>$.

Siqueira, J. T. T., \& Teixeira, M. J. (2001). Dor orofacial: diagnóstico, terapêutica e qualidade de vida. Curitiba: Maio.

Straub, R. O. (2005). Psicologia da saúde. Porto Alegre: Artmed.

Vasconcellos, E. G. (1998). Tópicos de psiconeuroimunologia. São Paulo: Editorial Ipê.

Vasconcellos, E. G. (2000). Psiconeuroimunologia: uma história para o futuro. In V. A. Angerami-Camon (Org.), Psicologia da saúde: um novo significado para a prática clínica (pp.23-41). São Paulo: Pioneira.

Vasconcellos, E. G. (2002). O modelo psiconeuroendocrinológico de stress. In L. Seger (Org.), Psicologia e odontologia: uma abordagem integradora (pp.35-60). São Paulo: Livraria Santos.

Recebido em: 26/7/2011

Versão final em: 25/1/2012

Aprovado em: 9/3/2012 
